\title{
Smoothness property on parameters of periodic systems
}

\author{
Min He
}

Correspondence: mhe@kent.edu Kent State University at Trumbull, Warren, $\mathrm{OH}$ 44483, USA

\begin{abstract}
This work is concerned with periodic systems dependent on parameters and investigates differentiability with respect to parameters of the periodic solutions of the systems. Some challenging situations arise from a hyperbolic type of periodic boundary value problem. Using some auxiliary operators and applying semigroup theory and a fixed point theorem, we are able to handle these cases and obtain the results on the existence and differentiability with respect to parameters of periodic solutions. The application of the obtained abstract results to a periodic boundary value problem is discussed at the end of the article.
\end{abstract}

AMS Subject Classification: 47D62; 45K05; 35L20.

Keywords: $C_{0}$-semigroup, periodic system, parameter, differentiability

\section{Introduction}

Our recent work [1] studied the following periodic system dependent on parameter:

$$
\begin{aligned}
& \frac{d z(t)}{d t}=A(\varepsilon) z(t)+f(t, z(t), \varepsilon), \\
& z(0)=z_{0} .
\end{aligned}
$$

and obtained a set of results for the existence of periodic solutions and the differentiability with respect to parameter $\varepsilon$ of such solutions. Those results are effectively applied to parabolic type of periodic systems. The operator of such systems generates an analytic $C_{0}$-semigroup $T(t, \varepsilon)$, which possesses some nice properties such as (a) the $C_{0}$-semigroup $T(t, \varepsilon)$ satisfies the contraction condition; (b) the $C_{0}$-semigroup $T(t, \varepsilon)$ is differentiable with respect to parameter $\varepsilon$ on the entire space (see [2] for details). And these two properties are the key for determining the existence and differentiability with respect to parameter of the periodic solution. Hence, the conditions of the obtained theorems in [1] are proposed for determining that the system has these two properties.

However, we observe that some hyperbolic types of equations do not have the abovementioned properties. Take, for example, a wave equation with forced and damped boundary conditions:

$$
\begin{array}{lr}
u_{t t}=u_{x x} & \text { for } t \geq 0, \\
u(x, 0)=u_{0}(x), \quad u_{t}(x, 0)=u_{1}(x) & \text { for } x \in[0,1] \\
\mu u_{t}(0, t)-\gamma u_{x}(0, t)=f_{1}(t), & \\
\delta u_{t}(1, t)+\gamma u_{x}(1, t)=f_{2}(t), & \mu, \gamma, \delta>0,
\end{array}
$$


where $f_{1}(t)$ and $f_{2}(t)$ are both $\rho$-periodic and continuously differentiable. Notice that the boundary conditions contain three scalars $\mu, \gamma$, and $\delta$. These scalars are considered as parameters because they may vary as the environment of the system changes. Reforming (1.2) (see Section 5 for details), we have the following periodic boundary value problem:

$$
\begin{aligned}
& \bar{v}_{t}=\bar{w}_{x}+\gamma^{-1} f_{1}(t)-x \delta^{-1}{f^{\prime}}_{2}(t), \\
& \bar{w}_{t}=\bar{v}_{x}+\delta^{-1} f_{2}(t)+(1-x) \gamma^{-1}{f^{\prime}}_{1}(t), \\
& \mu \bar{v}(0, t)-\gamma \bar{w}(0, t)=0, \quad \mu, \gamma, \delta>0 . \\
& \delta \bar{v}(1, t)+\gamma \bar{w}(1, t)=0, \quad
\end{aligned}
$$

Further, the associated abstract equation of (1.3) is given by

$$
\begin{aligned}
& \frac{d z(t)}{d t}=A(\varepsilon) z(t)+F(t, \varepsilon), \\
& z(0)=z_{0}
\end{aligned}
$$

on $X=L^{2}[0,1] \times L^{2}[0,1]$, where

$$
\begin{aligned}
& A(\varepsilon)=\left[\begin{array}{cc}
0 & \partial x \\
\partial x & 0
\end{array}\right], \quad \varepsilon=(\mu, \gamma, \delta) \in R_{+}^{3}, \quad z=\left[\begin{array}{c}
\bar{v} \\
\bar{w}
\end{array}\right], \\
& D(A(\varepsilon))=\left\{\left[\begin{array}{c}
\bar{v} \\
\bar{w}
\end{array}\right] \in \prod_{i=1}^{2} H^{1}[0,1] \mid \begin{array}{c}
\mu \bar{v}(0)=\gamma \bar{w}(0), \\
\delta \bar{v}(1)=-\gamma \bar{w}(1),
\end{array}, \gamma, \delta>0\right\}, \\
& F(t, z, \varepsilon) x=\left[\begin{array}{c}
\gamma^{-1} f_{1}(t)-x \delta^{-1} f^{\prime}{ }_{2}(t) \\
\delta^{-1} f_{2}(t)+(1-x) \gamma^{-1} f^{\prime}{ }_{1}(t)
\end{array}\right] .
\end{aligned}
$$

By examining Equation 1.4, we see that (a) $F$ is a $\rho$-periodic function, (b) $\varepsilon$ is a multiparameter, (c) $A(\varepsilon)$ is linear and densely defined, and (d) the operator $A(\varepsilon)$ has a variable domain (i.e. the domain of the operator $D(A(\varepsilon))$ is dependent on the parameter $\varepsilon$ ). Furthermore, we notice that (a) the operator $A(\varepsilon)$ generates a $C_{0}$-semigroup $T(t, \varepsilon)$, which is not a contraction operator, but it becomes eventually contracting, that is $\| T$ $(N \rho, \varepsilon) \| \leq k<1$ for some integer $N$ with $N \rho<t_{0}$ and all $\varepsilon \in P$ (see Section 5 for details); (b) the existing results on differentiability with respect to parameters of nonanalytic $C_{0}$-semigroup are not on the entire space [3]. It is not easy to obtain the smoothness property on the entire space.

Being motivated by this example, we investigate these challenging and interesting phenomena and aim for obtaining the existence and differentiability with respect to parameter of the periodic solution of (1.1). In order to treat the situation that $T(t, \varepsilon)$ is not a contraction operator (but it becomes eventually contracting), we construct some auxiliary operator and show that it is contracting. With some suitable restriction on $f$, we can weaken the condition about smoothness property of results obtained in [1], and just require the differentiability on the domain of the operator. Further, by applying a fixed point theorem, we are able to show that the periodic system (1.1) has a unique periodic solution and it is (Frechét) differentiable with respect to parameter.

The semigroup theory indicates that, when $A(\varepsilon)$ generates a $C_{0}$-semigroup $T(t, \varepsilon)$, the weak solution of (1.1) can be expressed in terms of the $C_{0}$-semigroup $T(t, \varepsilon)$ :

$$
z(t, \varepsilon)=T(t, \varepsilon) z_{0}+\int_{0}^{t} T(t-s, \varepsilon) f(s, z(s, \varepsilon), \varepsilon) d s .
$$


Clearly, the differentiability with respect to parameter $\varepsilon$ of the $C_{0}$-semigroup $T(t, \varepsilon)$ is critical in determining the differentiability with respect to parameter $\varepsilon$ of the solution $z$ $(t, \varepsilon)$ of $(1.1)$. Hence, our previous works [2,3], and reference therein have focused on the differentiability with respect to parameter $\varepsilon$ of the semigroup $T(t, \varepsilon)$. A series of results have been obtained on this topic. Especially, some of these results are very conveniently and effectively applied to the case of the variable domain of the operator. Thus, in this article, we can just assume that the $C_{0}$-semigroup $T(t, \varepsilon)$ has such smoothness property and apply some of these existing results to prove the differentiability with respect to parameter $\varepsilon$ of the periodic solution of (1.1).

This article is organized as follows. In Section 2, we state some differentiability result, a fixed point theorem, and some related theorems. These results will be used in later sections. In Section 3, we study a special case of Equation 1.1

$$
\begin{aligned}
& z^{\prime}=A(\varepsilon) z+f(t, \varepsilon), \\
& z(0)=z_{0},
\end{aligned}
$$

where $f(t+\rho, \varepsilon)=f(t, \varepsilon)$ for some $\rho>0$, and $f(t, \varepsilon)$ is continuous in $(t, \varepsilon) \in R \times P$. Using an auxiliary operator and apply a fixed point theorem, we are able to prove that (1.5) has a unique periodic solution, and it is continuously (Frechét) differentiable with respect to parameter. In Section 4, we will use the fixed point theorem as a tool and apply the results obtained from Section 3 to obtain the desired results for Equation 1.1. In Section 5, we will discuss the application of obtained abstract results to periodic boundary value problem (1.2) and use this example to illustrate the obtained abstract results.

\section{Preliminaries}

In this section, we present some existing theorems that will be used in later proofs. We start by giving a result on differentiability with respect to parameters.

Consider the abstract Cauchy problem (1.1) where $A(\varepsilon)$ is a closed linear operator on a Banach space $(\mathrm{X},\|\cdot\|)$ and $\varepsilon \in P$ is a multiparameter ( $P$ is an open subset of a finite-dimensional normed linear space with norm $|\cdot|)$. Let $T(t, \varepsilon)$ be the $C_{0}$-semigroup generated by the operator $A(\varepsilon)$. For further information on $C_{0}$-semigroup, see [4].

Assumption $\mathbf{Q}$. Let $\varepsilon_{0} \in P$ be given. Then, for each $\varepsilon \in P$, there exist bounded operators $Q_{1}(\varepsilon), Q_{2}(\varepsilon): X \rightarrow X$ with bounded inverses $Q_{1}^{-1}(\varepsilon)$ and $Q_{2}^{-1}(\varepsilon)$, such that $A$ $(\varepsilon)=Q_{1}(\varepsilon) A\left(\varepsilon_{0}\right) Q_{2}(\varepsilon)$.

Note that if $A\left(\varepsilon_{1}\right)=Q_{1}\left(\varepsilon_{1}\right) A\left(\varepsilon_{0}\right) Q_{2}\left(\varepsilon_{1}\right)$, then

$$
\begin{aligned}
A(\varepsilon) & =Q_{1}(\varepsilon) A\left(\varepsilon_{0}\right) Q_{2}(\varepsilon) \\
& =Q_{1}(\varepsilon) Q_{2}^{-1}\left(\varepsilon_{1}\right) Q_{1}\left(\varepsilon_{1}\right) A\left(\varepsilon_{0}\right) Q_{2}\left(\varepsilon_{1}\right) Q_{2}^{-1}\left(\varepsilon_{1}\right) Q_{2}(\varepsilon) \\
& =\tilde{Q}_{1}(\varepsilon) A\left(\varepsilon_{1}\right) \tilde{Q}_{2}(\varepsilon) .
\end{aligned}
$$

Thus, having such a relationship for some $\varepsilon_{0}$ implies a similar relationship at any other $\varepsilon_{1} \in P$. Without loss of generality then, we may just consider the differentiability of the semigroup $T(t, \varepsilon)$ at $\varepsilon=\varepsilon_{0} \in P$. In the sequel, we consider all differentiability at $\varepsilon=\varepsilon_{0} \in P$.

Theorem 2.1. [3] Assume Assumption $Q$ and that 
(2.1) $Q_{i}(\varepsilon) x$ and $Q_{i}^{-1}(\varepsilon) x$ are continuously (Frechét) differentiable with respect to $\varepsilon$ for $x \in X, i=1$, 2. Then, for each $x \in D\left(A\left(\varepsilon_{0}\right)\right)$, the $C_{0}$-semigroup $T(t, \varepsilon) x$ is continuously (Frechét) differentiable at $\varepsilon=\varepsilon_{0}$ for $\varepsilon_{0} \in P$. In particular,

$$
\begin{aligned}
{\left[\left.D_{\varepsilon} T(t, \varepsilon) x\right|_{\varepsilon=\varepsilon_{0}}\right]=[} & \left.\left.D_{\varepsilon} Q_{2}^{-1}(\varepsilon) T\left(t, \varepsilon_{0}\right) x\right|_{\varepsilon=\varepsilon_{0}}\right]+Q_{2}^{-1}\left(\varepsilon_{0}\right)\left[\left.D_{\varepsilon} T(t, \varepsilon) x\right|_{\varepsilon=\varepsilon_{0}}\right] \\
& +Q_{2}^{-1}\left(\varepsilon_{0}\right) T\left(t, \varepsilon_{0}\right)\left[\left.D_{\varepsilon} Q_{2}(\varepsilon) x\right|_{\varepsilon=\varepsilon_{0}}\right] .
\end{aligned}
$$

Theorem 2.2. [5] Assume Assumption $Q$ and that

(2.2) there are constants $M \geq 1$ and $\omega \in R$ such that

$$
\left\|(\lambda I-A(\varepsilon))^{-n}\right\| \leq \frac{M}{(\lambda-\omega)^{n}} \text { for } \lambda>\omega, n \in N \text { and all } \varepsilon \in P .
$$

(2.3) $Q_{i}(\varepsilon) x$ and $Q_{2}^{-1}(\varepsilon) x$ are continuous in $\varepsilon$ for $x \in X, i=1,2$.

Then, $C_{0}$-semigroup $T(t, \varepsilon)$ generated by $A(\varepsilon)$ is strongly continuous at $\varepsilon_{0}$, and the continuity is uniform on bounded $t$-intervals. In particular, for any $h \in \mathcal{P}$ with $\varepsilon_{0}+h$ $\in P$, and any $t_{0} \in[0, \infty)$,

$\sup _{0 \leq t \leq t_{0}}\left\|T\left(t, \varepsilon_{0}+h\right) x-T\left(t, \varepsilon_{0}\right) x\right\|=\mathrm{o}(1)$ as $|h| \rightarrow 0$ for each $x \in X$.

Now, we state a fixed point theorem from [6].

Definition 2.3. [6, p. 6] Suppose $\mathcal{F}$ is a subset of a Banach space $(\mathcal{X},|\cdot|), \mathcal{G}$ is a subset of a Banach space $\mathcal{Y}$, and $\left\{T_{\gamma}, y \in \mathcal{G}\right\}$ is a family of operators taking $\mathcal{F} \rightarrow \mathcal{X}$. The operator $T_{y}$ is said to be a uniform contraction on $\mathcal{F}$ if $T_{y}: \mathcal{F} \rightarrow \mathcal{F}$, and there is a $\lambda, 0 \leq \lambda<1$ such that

$$
\left|T_{\gamma} x-T_{\gamma} \bar{x}\right| \leq \lambda|x-\bar{x}| \quad \text { for all } y \text { in } \mathcal{G}, x, \bar{x} \text { in } \mathcal{F}
$$

Theorem 2.4. [6, p. 7] If $\mathcal{F}$ is a closed subset of a Banach space $\mathcal{X}, \mathcal{G}$ is a subset of a Banach space $\mathcal{Y}, T_{y}: \mathcal{F} \rightarrow \mathcal{F}, \dagger$ in $\mathcal{G}$ is a uniform contraction on $\mathcal{F}$, and $T_{\mathrm{y}} x$ is continuous in $y$ for each fixed $x$ in $\mathcal{F}$, then the unique fixed point $g(y)$ of $T_{y}, y$ in $\mathcal{G}$, is continuous in $y$. Furthermore, if $\mathcal{F}, \mathcal{G}$ are the closures of open sets $\mathcal{F}^{\circ}, \mathcal{G}^{\circ}$, and $T_{y} x$ has continuous first derivatives $A(x, y), B(x, y)$ in $y, x$, respectively, for $x \in \mathcal{F}^{\circ}, \dagger \in \mathcal{G}^{\circ}$, then $g(y)$ has a continuous first derivative with respect to $y$ in $\mathcal{G}^{\circ}$.

Theorem 2.5. [7, p. 167] Let $f$ be a continuous mapping of an open subset $\Omega$ of $E$ into $F$. $f$ is continuously (Frechét) differentiable in $\Omega$ iff $f$ is (Frechét) differentiable at each point with respect to the $i$ th $(i=1,2, \ldots, n)$ variable, and the mapping $\left(x_{1}, \ldots, x_{n}\right) \rightarrow D_{i} f$ $\left(x_{1}, \ldots, x_{n}\right)$ (of $\Omega$ into $\left.\mathcal{B}\left(\varepsilon_{\gamma}, \mathcal{F}\right)\right)$ ) is continuous in $\Omega$. Then, at each point $\left(x_{1}, \ldots, x_{n}\right)$ of $\Omega$, the derivative of $f$ is given by

$$
D f\left(x_{1}, \ldots, x_{n}\right) \cdot\left(t_{1}, \ldots, t_{n}\right)=\sum_{i=1}^{n} D_{i} f\left(x_{1}, \ldots, x_{n}\right) \cdot t_{i}, \quad\left(t_{1}, \ldots, t_{n}\right) \in E .
$$

Lemma 2.6. Let $B \in \mathcal{B}(\mathcal{X}, \mathcal{Y})$. If $\|B\| \leq \alpha$, then $(I-B)^{-1}$ exists, and

$$
(I-B)^{-1}=\sum_{k=0}^{\infty} B^{k}
$$

Moreover, $\left\|(I-B)^{-1}\right\| \leq \frac{1}{1-\alpha}$. 
Proof. The proof is standard and is omitted here.

\section{Results on Equation 1.5}

The goal of this section is to obtain the existence and differentiability with respect to parameter of the unique periodic solution of (1.5), which is a special case of Equation 1.1. As noted in Section 2, without loss of generality, we may just consider the differentiability of semigroup $T(t, \varepsilon)$ at $\varepsilon=\varepsilon_{0} \in P$. Also, Theorem 2.1 illustrates some convenient conditions for determining differentiability with respect to parameter of $C_{0}$-semigroup $T(t, \varepsilon)$ on the domain of the operator. Thereby, in this section, we will assume that $T(t, \varepsilon)$ holds the smoothness property with respect to parameter $\varepsilon$.

According to the semigroup theory, when $A(\varepsilon)$ generates a $C_{0}$-semigroup $T(t, \varepsilon)$, the weak solution of (1.5) can be expressed in terms of the $C_{0}$-semigroup $T(t, \varepsilon)$ :

$$
z(t, \varepsilon)=T(t, \varepsilon) z_{0}+\int_{0}^{t} T(t-s, \varepsilon) f(s, \varepsilon) d s
$$

To show that there is a unique $\rho$-periodic solution which is continuously (Frechét) differentiable with respect to parameter $\varepsilon$, it suffices to show that the operator $K(\varepsilon)$ has a unique fixed point with smoothness property with respect to parameter $\varepsilon$ where

$$
K(\varepsilon) z=T(\rho, \varepsilon) z+\int_{0}^{\rho} T(\rho-s, \varepsilon) f(s, \varepsilon) d s .
$$

As noted in the Introduction section, the example (Equation 1.2) suggests that the $C_{0^{-}}$-semigroup $T(t, \varepsilon)$ is not contracting, but it is eventually contracting, that is $\|T(N \rho, \varepsilon)\|$ $\leq k<1$ for some integer $N$ with $N \rho<t_{0}$ and all $\varepsilon \in P$ (see Section 5 for details).

To handle this situation, we consider the $N$ th-iterate, $K^{N}(\varepsilon)$. We first note that $K^{N}(\varepsilon)$ is a uniform contraction on $X$. In fact, note that

$$
K^{N}(\varepsilon) z=T(N \rho, \varepsilon) z+\int_{0}^{N \rho} T(N \rho-s, \varepsilon) f(s, \varepsilon) d s
$$

and then, for all $\varepsilon \in P$ and $z_{1}, z_{2} \in X$,

$$
\begin{aligned}
& \left\|K^{N}(\varepsilon) z_{1}-K^{N}(\varepsilon) z_{2}\right\|=\left\|T(N \rho, \varepsilon)\left(z_{1}-z_{2}\right)\right\| \\
\leq \quad & \|T(N \rho, \varepsilon)\| \cdot\left\|z_{1}-z_{2}\right\| \leq k\left\|z_{1}-z_{2}\right\| \quad(\text { since }\|T(N \rho, \varepsilon)\| \leq k<1) .
\end{aligned}
$$

Further, if the operator $K^{N}(\varepsilon)$ has a fixed point, say $z_{0}(\varepsilon)$ with the smoothness property with respect to $\varepsilon$, then $z_{0}(\varepsilon)$ is also the fixed point of $K(\varepsilon)$. To this end, we just need to show that the operator $K^{N}(\varepsilon)$ has a fixed point with the smoothness property with respect to parameter $\varepsilon$.

We want to apply the fixed point theorem (Theorem 2.4) to obtain the desired result. Let us begin with the following lemma.

Lemma 3.1. Assume that

(3.1) $T(t, \varepsilon) z$ is continuous in $\varepsilon$ for each $z \in X$, and $T(t, \varepsilon) z$ is continuously (Frechét) differentiable with respect to $\varepsilon$ for each $z \in D\left(A\left(\varepsilon_{0}\right)\right)$.

(3.2) $f(t, \varepsilon)$ is continuously (Frechét) differentiable with respect to $\varepsilon$ and $f(t, \varepsilon) \in D\left(A\left(\varepsilon_{0}\right)\right)$. Then,

(a) $T(t-s, \varepsilon) f(s, \varepsilon)$ is continuously (Frechét) differentiable with respect to $\varepsilon$ for $t-s$, $s \in[0, \rho]$. In particular, for any $\varepsilon_{0} \in P$, 


$$
\begin{gathered}
{\left.\left[D_{\varepsilon} T(t-s, \varepsilon) f(s, \varepsilon)\right]\right|_{\varepsilon=\varepsilon_{0}}} \\
=\left.\quad\left[D_{\varepsilon} T(t-s, \varepsilon) f\left(s, \varepsilon_{0}\right)\right]\right|_{\varepsilon=\varepsilon_{0}}+\left.T\left(t-s, \varepsilon_{0}\right)\left[D_{\varepsilon} f(s, \varepsilon)\right]\right|_{\varepsilon=\varepsilon_{0}} .
\end{gathered}
$$

(b) $D_{\varepsilon}\left[\int_{0}^{\rho} T(N \rho-s, \varepsilon) f(s, \varepsilon) d s\right]=\int_{0}^{\rho}\left[D_{\varepsilon} T(N \rho-s, \varepsilon) f(s, \varepsilon)\right] d s$.

Proof. The proof of Part (a) is similar to the argument in [1, Theorem 3.4] and is omitted here.

To prove Part (b), according to Theorem 2.5, it suffices to show that

$$
D_{\varepsilon i}\left[\int_{0}^{\rho} T(N \rho-s, \varepsilon) f(s, \varepsilon) d s\right]=\int_{0}^{\rho}\left[D_{\varepsilon i} T(N \rho-s, \varepsilon) f(s, \varepsilon)\right] d s \quad(i=1, \ldots, n) .
$$

The rest of the proof is similar to the argument in [1, Theorem 3.4] and is also omitted here.

Theorem 3.2. Assume that (3.1) and (3.2) are satisfied and support that

(3.3) $\|T(N \rho, \varepsilon)\| \leq k<1$ for some integer $N$ with $N \rho<t_{0}$ and all $\varepsilon \in P$.

Then, there exists a unique $\rho$-periodic solution of (1.5), say $z(t, \varepsilon)$, which is continuously (Frechét) differentiable with respect to $\varepsilon$ for $\varepsilon \in P$.

Proof. We will apply the fixed point theorem-Theorem 2.4 to show that the operator $K^{N}(\varepsilon)$ has $z_{0}(\varepsilon)$ as the fixed point, and $z_{0}(\varepsilon)$ is continuously (Frechét) differentiable with respect to $\varepsilon$.

Note that the operator $K^{N}(\varepsilon)$ has the following properties.

(i) $K^{N}(\varepsilon)$ is defined on the Banach space $(X,\|\cdot\|)$.

(ii) $K^{N}(\varepsilon)$ is a uniform contraction on $X$.

In fact, for all $\varepsilon \in P$ and $z_{1}, z_{2} \in X$,

$$
\begin{aligned}
& \left\|K^{N}(\varepsilon) z_{1}-K^{N}(\varepsilon) z_{2}\right\|=\left\|T(N \rho, \varepsilon)\left(z_{1}-z_{2}\right)\right\| \\
\leq \quad & \|T(N \rho, \varepsilon)\| \cdot\left\|z_{1}-z_{2}\right\| \leq k\left\|z_{1}-z_{2}\right\| \quad(\text { since }\|T(N \rho, \varepsilon)\| \leq k<1) .
\end{aligned}
$$

(iii) $K^{N}(\varepsilon) z$ is continuous in $\varepsilon$ for each fixed $z \in X$. (For the detailed proof, see Theorem 3.2 from [5].)

Applying Theorem 2.4, we have that $z_{0}(\varepsilon)$ is the fixed point of $K^{N}(\varepsilon)$.

Further, it is clear that the first derivative of $K^{N}(\varepsilon) z$ with respect to $z$

$$
D_{z} K^{N}(\varepsilon)_{Z}=T(N \rho, \varepsilon)
$$

is continuous in $\varepsilon$. It is also clear from Lemma 3.1 that the first derivative of $K^{N}(\varepsilon) z$ with respect to $\varepsilon$ has the form

$$
\left[D_{\varepsilon} K^{N}(\varepsilon) z\right]=D_{\varepsilon}[T(N \rho, \varepsilon) z]+\int_{0}^{\rho}\left[D_{\varepsilon} T(N \rho-s, \varepsilon) f(s, \varepsilon)\right] d s .
$$

By Assumptions (3.1) and (3.2) we can use the similar argument as that in Lemma 3.1-Part (a) to show that $\left[D_{\varepsilon} T(\rho-s, \varepsilon) f(s, \varepsilon)\right]$ is continuous at $\varepsilon=\varepsilon_{0}$ and thereby $\int_{0}^{\rho}\left[D_{\varepsilon} T(\rho-s, \varepsilon) f(s, \varepsilon)\right] d s$ is continuous at $\varepsilon_{0}$.

Thus, $\left[D_{\varepsilon} K^{N}(\varepsilon) z\right]$ is continuous in $(z, \varepsilon)$.

Now, all conditions of Theorem 2.4 are satisfied. It follows from Theorem 2.4 that there exists a fixed point $z_{0}(\varepsilon)$ of operator $K^{N}(\varepsilon)$, which is continuously (Frechét) 
differentiable with respect to $\varepsilon$. As noted at the beginning of the section, $z_{0}(\varepsilon)$ is the fixed point of operator $K(\varepsilon)$.

Finally, using a similar argument as that in Lemma 3.1, we can show that $T(t, \varepsilon) z_{0}(\varepsilon)$ and $\int_{0}^{t} T(t-s, \varepsilon) f(s, \varepsilon) d s$ are continuously (Frechét) differentiable with respect to $\varepsilon$. Thus,

$$
z(t, \varepsilon)=T(t, \varepsilon) z_{0}(\varepsilon)+\int_{0}^{t} T(t-s, \varepsilon) f(s, \varepsilon) d s
$$

is continuously (Frechét) differentiable with respect to $\varepsilon$ for $\varepsilon \in P$.

Now, we present a theorem that is convenient in application since some assumption is posed on the operator $A(\varepsilon)$ instead of the $C_{0}$-semigroup $T(t, \varepsilon)$.

Theorem 3.3. Assume that Assumption Q, (3.2) and (3.3) are satisfied and suppose that

(3.4) $Q_{i}(\varepsilon) x$ and $Q_{i}^{-1}(\varepsilon) x$ are continuously (Frechét) differentiable with respect to $\varepsilon$ for $x \in X, i=1,2$.

Then, there exists a unique $\rho$-periodic solution of (1.5), say $z(t, \varepsilon)$, which is continuous in $\varepsilon$ for $\varepsilon \in P$.

Proof. This is an immediate result from Theorems 2.1, 2.2, and 3.2.

\section{Results on Equation 1.1}

In this section, we will discuss the general Equation 1.1

$$
\begin{aligned}
& \frac{d z(t)}{d t}=A(\varepsilon) z(t)+f(t, z(t), \varepsilon), \\
& z(0)=z_{0} .
\end{aligned}
$$

With the aid of semigroup theory, we are able to construct suitable operators. Applying a fixed point theorem, we obtain the existence and differentiability with respect to parameter of the unique periodic solution of (1.1).

Lemma 4.1. Assume that (3.1) and (3.3) are satisfied. Then, $(I-T(N \rho, \varepsilon))^{-1} z$ is continuously (Frechét) differentiable with respect to $\varepsilon$ for each $z \in D\left(A\left(\varepsilon_{0}\right)\right)$.

Proof. First note that from (3.3), we see that $(I-T(N \rho, \varepsilon))^{-1}$ exists by Lemma 2.6. Also,

$$
\left\|(I-T(N \rho, \varepsilon))^{-1}\right\| \leq \frac{1}{1-k} \doteq H .
$$

Next consider the operator defined on $X$ :

$J(\varepsilon) z=T(N \rho, \varepsilon) z+y$ where $y$ is a given point in $X$.

Then, we have

$$
\left\|J(\varepsilon) z_{1}-J(\varepsilon) z_{2}\right\| \leq\|T(N \rho, \varepsilon)\| \cdot\left\|z_{1}-z_{2}\right\| \leq k\left\|z_{1}-z_{2}\right\|,
$$

so $J(\varepsilon)$ is a uniform contraction. Also, it is obvious that $J(\varepsilon) z$ is continuous in $\varepsilon$ by (3.1). Therefore, from Theorem 2.4, it follows that there is a unique fixed point of $J(\varepsilon)$, say $z(\varepsilon)$.

Furthermore, since

$$
\begin{aligned}
& D_{\varepsilon} J(\varepsilon) z=D_{\varepsilon} T(N \rho, \varepsilon) z \text { for } z \in D\left(a\left(\varepsilon_{0}\right)\right), \\
& D_{z} J(\varepsilon) z=T(N \rho, \varepsilon),
\end{aligned}
$$


which clearly satisfy the conditions of Theorem 2.4 , so by Theorem 2.4 we have

$$
z(\varepsilon)=(I-T(N \rho, \varepsilon))^{-1} \gamma
$$

is continuously (Frechét) differentiable with respect to $\varepsilon$.

Let $P C[R, \rho]=\{g \in C(R) \mid g(t+\rho)=g(t)\}$.

Consider the equation

$$
\begin{aligned}
& z(t)^{\prime}=A(\varepsilon) z(t)+f(t, g(t), \varepsilon), \\
& z(0)=z_{0}
\end{aligned}
$$

on a Banach space $(X,\|\cdot\|)$, where $f(t+\rho, g, \varepsilon)=f(t, g, \varepsilon)$ for some $\rho>0$ and $g \in P C$ $[R, \rho]$, and $f(t, g, \varepsilon)$ is continuous in $(t, g, \varepsilon) \in R \times P C[R, \rho] \times P$.

Lemma 4.2. Assume that (3.3) is satisfied and suppose that

(4.3) $T(t, \varepsilon) z$ is continuous in $\varepsilon$ for $z \in X$, and it is continuously (Frechét) differentiable with respect to $\varepsilon$ for each $z \in D\left(A\left(\varepsilon_{0}\right)\right)$. Moreover, for any $\varepsilon_{0} L P$, there is some $\delta\left(\varepsilon_{0}\right)>0$ such that $\varepsilon \in B\left(\varepsilon_{0}, \delta\left(\varepsilon_{0}\right)\right)$

$$
\left\|D_{\varepsilon} T(t, \varepsilon) z\right\| \leq \bar{H}\left(\varepsilon_{0}\right)\|z\| \quad \text { for some } \bar{H}\left(\varepsilon_{0}\right)>0 \text { and } t \in[0, \rho] .
$$

(4.4) $f(t, z, \varepsilon)$ is continuously (Frechét) differentiable with respect to $\varepsilon$ and $f(t, z(t), \varepsilon) \in$ $D\left(A\left(\varepsilon_{0}\right)\right)$.

Then, there exists a unique $\rho$-periodic solution of (4.2), say $z(t, \varepsilon, g)$, which is continuously (Frechét) differentiable with respect to $\varepsilon$ for $\varepsilon \in P$. Also,

$$
z(0, \varepsilon, g)=(I-T(N \rho, \varepsilon))^{-1} \int_{0}^{\rho} T(N \rho-s, \varepsilon) f(s, g(s), \varepsilon) d s,
$$

which is continuously (Frechét) differentiable with respect to $\varepsilon$.

Proof. Let $F(t, \varepsilon)=f(t, g(t), \varepsilon)$. Then, $F(t+\rho, \varepsilon)=F(t, \varepsilon)$. Also, it is obvious that $F(t, \varepsilon)$ satisfies (3.2). Equations 4.3 and 4.4 indicate other conditions of Theorem 3.2 are satisfied. Therefore, it follows from Theorem 3.2 that there is a unique $\rho$-solution $z(t, \varepsilon, g)$ of Equation 4.2, which is continuously (Frechét) differentiable with respect to $\varepsilon$. In particular, $z(0, \varepsilon, g)$ is continuously (Frechét) differentiable with respect to $\varepsilon$. Moreover, using the same argument as that in the proof of Theorem 3.2, we see that

$$
z(0, \varepsilon, g)=T(N \rho, \varepsilon) z(0, \varepsilon, g)+\int_{0}^{\rho} T(N \rho-s, \varepsilon) f(s, g(s), \varepsilon) d s .
$$

Thus,

$$
z(0, \varepsilon, g)=(I-T(N \rho, \varepsilon))^{-1} \int_{0}^{\rho} T(N \rho-s, \varepsilon) f(s, g(s), \varepsilon) d s,
$$

which is continuously (Frechét) differentiable with respect to $\varepsilon$ by Lemma 4.1.

Define $J_{1}(\varepsilon): P C[R, \rho] \rightarrow P C[R, \rho]$ by

$$
J_{1}(\varepsilon) g(t)=T(t, \varepsilon) z(0, \varepsilon, g)+\int_{0}^{t} T(t-s, \varepsilon) f(s, g(s), \varepsilon) d s .
$$

Lemma 4.3. Assume (3.3) and that 
(4.5) $T(t, \varepsilon) z$ is continuous in $\varepsilon$ for $z \in X$, and

$$
\|T(t, \varepsilon)\| \leq M\left(t_{0}\right)
$$

for some $M\left(t_{0}>0\right)$ and all $\varepsilon \in P, t \in\left[0, t_{0}\right]$.

(4.6) $\left\|f\left(t, z_{1}, \varepsilon\right)-f\left(t, z_{2}, \varepsilon\right)\right\| \leq L(\varepsilon)\left\|z_{1}-z_{2}\right\|$, where $L(\varepsilon)$ is continuous in $\varepsilon \in P$ and $L(0)$ $=0$.

(4.7) $f_{2}(t, g, \varepsilon)=\frac{\partial}{\partial g} f(t, g, \varepsilon)$ is continuous in $(t, g, \varepsilon)$.

Then, the operator $J_{1}(\varepsilon)$ has a unique fixed point $g(\cdot, \varepsilon) \in P C[R, \rho]$ which is continuously (Frechét) differentiable with respect to $\varepsilon$.

Proof. First note that it is clear that $\left(P C[R, \rho],\|\cdot\|_{\infty}\right)$ is a Banach space. Next, since $L(0)=0$, then, by continuity of $L\left(\varepsilon^{1}\right)$, there is $\delta_{0}$ such that $\left|\varepsilon^{1}\right|<\delta_{0}$ implies

$$
L\left(\varepsilon^{1}\right) \leq \frac{1}{4} \min \left\{\frac{1}{H M^{2}\left(t_{0}\right) t_{0}}, \frac{1}{M\left(t_{0}\right) t_{0}}\right\} .
$$

Now, for $\varepsilon \in P$ with $\left|\varepsilon^{1}\right|<\delta_{0}$,

$$
\begin{aligned}
& \|T(t, \varepsilon)\| \cdot\left\|z\left(0, g_{1}, \varepsilon\right)-z\left(0, g_{2}, \varepsilon\right)\right\| \\
= & \|T(t, \varepsilon)\| \cdot\left\|(I-T(N \rho, \varepsilon))^{-1} \int_{0}^{N \rho} T(N \rho-s, \varepsilon)\left[f\left(s, g_{1}, \varepsilon\right)-f\left(s, g_{2}, \varepsilon\right)\right] d s\right\| \\
\leq & M\left(t_{0}\right)\left\|(I-T(N \rho, \varepsilon))^{-1}\right\| \int_{0}^{N \rho} \mid T(N \rho-s, \varepsilon)\|\cdot\| f\left(s, g_{1}, \varepsilon\right)-f\left(s, g_{2}, \varepsilon\right) \| d s \\
\leq & M\left(t_{0}\right) \cdot H \cdot M\left(t_{0}\right) \int_{0}^{N \rho}\left\|f\left(s, g_{1}, \varepsilon\right)-f\left(s, g_{2}, \varepsilon\right)\right\| d s \\
\leq & H \cdot M^{2}\left(t_{0}\right) \cdot t_{0} \cdot L\left(\varepsilon^{1}\right)\left\|g_{1}-g_{2}\right\| \leq \frac{1}{4}\left\|g_{1}-g_{2}\right\|
\end{aligned}
$$

and

$$
\begin{aligned}
& \int_{0}^{t}\|T(t-s, \varepsilon)\| \cdot\left\|f\left(s, g_{1}(s), \varepsilon\right)-f\left(s, g_{2}(s), \varepsilon\right)\right\| d s \\
\leq & M\left(t_{0}\right) L\left(\varepsilon^{1}\right) \int_{0}^{t}\left\|g_{1}(s)-g_{2}(s)\right\| d s \\
\leq & M\left(t_{0}\right) \cdot t_{0} L\left(\varepsilon^{1}\right)\left\|g_{1}-g_{2}\right\| \leq \frac{1}{4}\left\|g_{1}-g_{2}\right\| .
\end{aligned}
$$

Hence,

$$
\begin{aligned}
& \left\|J_{1}(\varepsilon) g_{1}-J_{1}(\varepsilon) g_{2}\right\| \\
\leq & \|T(t, \varepsilon)\| \cdot\left\|z\left(0, g_{1}, \varepsilon\right)-z\left(0, g_{2}, \varepsilon\right)\right\|+\int_{0}^{t}\|T(t-s, \varepsilon)\| \cdot \|\left(f\left(s, g_{1}(s), \varepsilon\right)-f\left(s, g_{2}(s), \varepsilon\right) \| d s\right. \\
\leq & \frac{1}{4}\left\|g_{1}-g_{2}\right\|+\frac{1}{4}\left\|g_{1}-g_{2}\right\| \\
\leq & \frac{1}{2}\left\|g_{1}-g_{2}\right\| .
\end{aligned}
$$

Therefore, $J_{1}(\varepsilon)$ is a uniform contraction.

Furthermore, $J_{1}(\varepsilon) g$ is continuous in $\varepsilon$ for fixed $g$. Using the similar argument as that in the proof of [5, Theorem 2.3], we have 


$$
\begin{aligned}
& D_{g} J_{1}(\varepsilon) g=T(t, \varepsilon) D_{g} z(0, \varepsilon, g)+\int_{0}^{t} T(t-s, \varepsilon) f_{2}(s, g(s), \varepsilon) d s \\
& \quad=T(t, \varepsilon)(I-T(\rho, \varepsilon))^{-1} \int_{0}^{\rho} f_{2}(s, g(s), \varepsilon) d s+\int_{0}^{t} T(t-s, \varepsilon) f_{2}(s, g(s), \varepsilon) d s, \\
& D_{\varepsilon} J_{1}(\varepsilon) g=\left[D_{\varepsilon} T(t, \varepsilon) z(0, \varepsilon, g)\right]+\int_{0}^{t}\left[D_{\varepsilon} T(t-s, \varepsilon) f(s, g(s), \varepsilon)\right] d s
\end{aligned}
$$

are continuous in $(g, \varepsilon)$. Therefore, from Theorem 2.4, it follows that $J_{1}(\varepsilon)$ has a unique fixed point, say $g(\cdot, \varepsilon) \in P C[R, \rho]$, which is continuously (Frechét) differentiable with respect to $\varepsilon$.

Now, we present the main theorem for Equation 1.1.

Theorem 4.4. Assume that (3.3) and (4.3)-(4.7) are satisfied. Then, there exists a unique $\rho$-periodic solution of (1.1), say $z(t, \varepsilon)$, which is continuously (Frechét) differentiable with respect to $\varepsilon$ for $\varepsilon \in P$.

Proof. This is an immediate result from Lemmas 4.2 and 4.3.

\section{Application to a periodic boundary value problem}

Consider the periodic boundary value problem:

$$
\begin{array}{lc}
u_{t t}=u_{x x} & \text { for } t \geq 0, \\
u(x, 0)=u_{0}(x), \quad u_{t}(x, 0)=u_{1}(x) \text { for } x \in[0,1] \\
\mu u_{t}(0, t)-\gamma u_{x}(0, t)=f_{1}(t), & \\
\delta u_{t}(1, t)+\gamma u_{x}(1, t)=f_{2}(t), & \mu, \gamma, \delta>0,
\end{array}
$$

where $f_{1}(t)$ and $f_{2}(t)$ are both $\rho$-periodic and continuously differentiable.

Let $v=u_{t}$ and $w=u_{x}$, then Equation 5.1 is written as

$$
\begin{aligned}
& v_{t}=w_{x} \\
& w_{t}=v_{x \prime} \\
& \mu v(0, t)-\gamma w(0, t)=f_{1}(t) \\
& \delta v(1, t)+\gamma w(1, t)=f_{2}(t), \quad \mu, \gamma, \delta>0 .
\end{aligned}
$$

If the change of variables

$$
\begin{aligned}
& \bar{v}=v-x \delta^{-1} f_{2} \\
& \bar{w}=w+(1-x) \gamma^{-1} f_{1}
\end{aligned}
$$

is made, Equation 5.1 has the form:

$$
\begin{aligned}
& \bar{v}_{t}=\bar{w}_{x}+\gamma^{-1} f_{1}(t)-x \delta^{-1} f_{2}^{\prime}(t), \\
& \bar{w}_{t}=\bar{v}_{x}+\delta^{-1} f_{2}(t)+(1-x) \gamma^{-1} f^{\prime}{ }_{1}(t), \\
& \mu \bar{v}(0, t)-\gamma \bar{w}(0, t)=0, \\
& \delta \bar{v}(1, t)+\gamma \bar{w}(1, t)=0, \quad \mu, \gamma, \delta>0 .
\end{aligned}
$$

Further, the associated abstract equation of (5.3) is given by

$$
\begin{aligned}
& \frac{d z(t)}{d t}=A(\varepsilon) z(t)+F(t, \varepsilon), \\
& z(0)=z_{0}
\end{aligned}
$$


on $X=L^{2}[0,1] \times L^{2}[0,1]$, where

$$
\begin{aligned}
& A(\varepsilon)=\left[\begin{array}{cc}
0 & \partial x \\
\partial x & 0
\end{array}\right], \quad \varepsilon=(\mu, \gamma, \delta) \in R_{+}^{3}, \quad z=\left[\begin{array}{l}
\bar{v} \\
\bar{w}
\end{array}\right], \\
& D(A(\varepsilon))=\left\{\left[\begin{array}{l}
\bar{v} \\
\bar{w}
\end{array}\right] \in \prod_{i=1}^{2} H^{1}[0,1] \mid \begin{array}{c}
\mu \bar{v}(0)=\gamma \bar{w}(0), \\
\delta \bar{v}(1)=-\gamma \bar{w}(1),
\end{array}, \gamma, \delta>0\right\}, \\
& F(t, z, \varepsilon) x=\left[\begin{array}{c}
\gamma^{-1} f_{1}(t)-x \delta^{-1} f^{\prime}{ }_{2}(t) \\
\delta^{-1} f_{2}(t)+(1-x) \gamma^{-1} f^{\prime}{ }_{1}(t)
\end{array}\right] .
\end{aligned}
$$

For convenience, write $\bar{v}$ as $v, \bar{w}$ as $w$, and $\alpha=\frac{\mu}{y}, \beta=\frac{y}{\delta}$, we then have

$$
\begin{aligned}
& \frac{d z(t)}{d t}=A(\varepsilon) z(t)+F(t, \varepsilon), \\
& z(0)=z_{0}
\end{aligned}
$$

on $X=L^{2}[0,1] \times L^{2}[0,1]$, where

$$
\begin{aligned}
& A(\varepsilon)=\left[\begin{array}{cc}
0 & \partial x \\
\partial x & 0
\end{array}\right], \quad \varepsilon=(\alpha, \beta, \delta) \in R_{+\prime}^{3} \quad z=\left[\begin{array}{c}
v \\
w
\end{array}\right], \\
& D(A(\varepsilon))=\left\{\left[\begin{array}{c}
v \\
w
\end{array}\right] \in \prod_{i=1}^{2} H^{1}[0,1] \begin{array}{c}
\alpha v(0)=w(0), \\
v(1)=-\beta w(1),
\end{array}, \beta, 0\right\}, \\
& F(t, \varepsilon) x=\left[\begin{array}{c}
\gamma^{-1} f_{1}(t)-x \delta^{-1} f^{\prime}(t) \\
\delta^{-1} f_{2}(t)+(1-x) \gamma^{-1} f^{\prime}{ }_{1}(t)
\end{array}\right] .
\end{aligned}
$$

Now, make the change of variables

$$
\tilde{z}=\left[\begin{array}{c}
\tilde{v} \\
\tilde{w}
\end{array}\right]=U\left[\begin{array}{c}
v \\
w
\end{array}\right] \text { where } \quad U=\frac{1}{2}\left[\begin{array}{cc}
1 & 1 \\
1 & -1
\end{array}\right]
$$

Then,

$$
A_{1}(\varepsilon) \equiv U A(\varepsilon) U^{-1}=\left[\begin{array}{cc}
\partial x & 0 \\
0 & -\partial x
\end{array}\right]
$$

where

$$
D\left(A_{1}(\varepsilon)\right)=\left\{\left[\begin{array}{c}
\tilde{v} \\
\tilde{w}
\end{array}\right] \in \prod_{i=1}^{2} H^{1}[0,1] \mid \begin{array}{c}
(1-\alpha) \tilde{v}(0)=(1+\alpha) \tilde{w}(0), \\
(1+\beta) \tilde{v}(1)=-(1-\beta) \tilde{w}(1) .
\end{array}\right\}
$$

Define

$$
\tilde{\alpha}=\frac{1-\alpha}{1+\alpha}, \quad \tilde{\beta}=\frac{1-\beta}{1+\beta} \quad \tilde{\alpha}, \tilde{\beta} \in(-1,1] .
$$

$A_{1}(\varepsilon)$ has the domain

$$
D\left(A_{1}(\varepsilon)\right)=\left\{\left[\begin{array}{c}
\tilde{v} \\
\tilde{w}
\end{array}\right] \in \prod_{i=1}^{2} H^{1}[0,1] \mid \begin{array}{c}
\tilde{a} \tilde{v}(0)=\tilde{w}(0), \\
\tilde{v}(1)=-\tilde{\beta} \tilde{w}(1),
\end{array}, \tilde{a} \in(-1,1)\right\} .
$$

If $|\tilde{\alpha} \tilde{\beta}|<1$, then $A_{1}(\varepsilon)$ generates a $C_{0}$-semigroup, $T_{1}(t, \varepsilon)$, on $X=L^{2}[0,1] \times L^{2}[0,1]$, endowed with the norm $\|(f, g)\| \equiv\|f\|_{2}+\|g\|_{2}$. It can easily be shown using the 
method of characteristics that this semigroup satisfies $\left\|T_{1}(t, \varepsilon)\right\|=1$ for $0 \leq t<1,\left\|T_{1}(t, \varepsilon)\right\|=\max \{|\tilde{\alpha}|,|\tilde{\beta}|\} \leq 1$ for $1 \leq t<2$, and $\left\|T_{1}(t, \varepsilon)\right\| \leq|\tilde{\alpha} \tilde{\beta}|<1$ for $t \geq 2$. Thus, the semigroup is eventually contracting.

It follows from $T(t, \varepsilon)=U T_{1}(t, \varepsilon) U^{-1}$ that $T(t, \varepsilon)$ must have the same properties with respect to the norm

$$
|\|z\|| \equiv\|U z\|
$$

on $X=L^{2}[0,1] \times L^{2}[0,1]$ when $\tilde{\alpha}, \tilde{\beta}>0$.

Now, consider the operator $A_{1}(\varepsilon)$. Take $\varepsilon_{0}=(0,0)$. For $\varepsilon=(\tilde{\alpha}, \tilde{\beta})$ with $\tilde{\alpha}, \tilde{\beta} \in(-1,1]$

$$
A_{1}(\varepsilon)=\frac{1}{1+\tilde{\alpha} \tilde{\beta}} Q(\varepsilon) A_{1}(0) Q(\varepsilon),
$$

where

$$
Q(\varepsilon)=\left[\begin{array}{cc}
1 & \tilde{\beta} \\
-\tilde{\alpha} & 1
\end{array}\right]
$$

obviously, $Q(\varepsilon)$ is continuously (Frechét) differentiable with respect to $\varepsilon$ and is bounded, and so is $Q^{-1}(\varepsilon)$. Let $Q_{2}(\varepsilon)=\frac{1}{1+\tilde{\alpha} \tilde{\beta}} Q(\varepsilon)$ and $\mathrm{Q}_{1}(\varepsilon)=Q(\varepsilon)$. It is clear that the hypotheses of Theorem 3.3 are satisfied for any $\varepsilon_{0}=\left(\tilde{\alpha}_{0}, \tilde{\beta}_{0}\right)$ in $R^{+} \times R^{+}$. That is, we have the (Frechét) differentiable with respect to parameter $\varepsilon$ of semigroup $T_{1}(t, \varepsilon)$ and thus the same property holds for semigroup $T(t, \varepsilon)$. Further, if we assume that $f_{1}^{\prime}(t)+f_{2}^{\prime}(t)=k f_{1}(t)$ where $k=\frac{\mu+\delta}{\gamma}$, then $F(t, \varepsilon) \in D\left(A\left(\varepsilon_{0}\right)\right)$.

Above all, all assumptions of Theorem 3.3 are satisfied. Thus, it follows from Theorem 3.3 that there is a unique $\rho$-periodic weak solution of (5.4) and thus also a unique $\rho$-periodic weak solution of (5.1) and it is $L^{2}$ continuously (Frechét) differentiable with respect to parameter $\varepsilon=(\alpha, \delta, \gamma)$.

\section{Authors' contributions}

Min $\mathrm{He}$ is the sole author of this manuscript.

\section{Competing interests}

The author declares that they have no competing interests.

Received: 5 May 2011 Accepted: 2 November 2011 Published: 2 November 2011

\section{References}

1. He, M: Periodic systems dependent on parameters. J Inequal Appl 17 (2010). Article ID 796165

2. He, M: A parameter dependence problem in parabolic PDEs. J Dyn Continuous Discrete Impulsive Syst 169-179 (2003). Ser. A 10

Grimmer, R, He, M: Differentiability with respect to parameters of semigroups. Semigroup Forum. 59(3), $317-333$ (1999)

Goldstein, J: Semigroups of Linear Operators and Applications. Oxford University Press, New York (1985)

Grimmer, R, He, M: Fixed point theory and nonlinear periodic systems. The CUBO Math J. 11(3), 101-104 (2009)

Hale, JK: Ordinary Differential Equations. Wiley-Interscience. New York (1969)

Dieudonné, J: Foundations of Modern Analysis. Academic Press, New York (1960) 